PROCEEDINGS OF THE

AMERICAN MATHEMATICAL SOCIETY

Volume 138, Number 9, September 2010, Pages 3105-3109

S 0002-9939(10)10401-8

Article electronically published on April 29, 2010

\title{
AFFINE VARIETIES WITH STABLY TRIVIAL ALGEBRAIC VECTOR BUNDLES
}

\author{
ZBIGNIEW JELONEK \\ (Communicated by Ted Chinburg)
}

\begin{abstract}
Let $k$ be an algebraically closed field. For every affine variety $X$ with $\operatorname{dim} X \geq 7$ we construct a smooth affine variety $Y$ which is birationally equivalent to $X$ and which possesses a stably trivial but not trivial algebraic vector bundle. We give some application of this fact to the cancellation problem.
\end{abstract}

\section{INTRODUCTION}

Let $k$ be an algebraically closed field and let $X$ be a smooth affine variety over $k$. If $\operatorname{dim} X \leq 2$, then there is no non-trivial and stably trivial algebraic vector bundle on it. Moreover, there are only few examples of such vector bundles in the general case (see 13, 14, 12). Here we deal with the question of whether such algebraic vector bundles can exist on a sufficiently general affine variety. We show that for every affine variety $X$ with $\operatorname{dim} X \geq 7$, there exists a smooth affine variety $Y$ which is birationally equivalent to $X$ and which possesses a stably trivial but not trivial algebraic vector bundle.

We apply this result to the cancellation problem. Let us recall that an affine variety $X$ has the cancellation property $(\mathrm{CP})$ if for every affine variety $Y$, if $X \times k \cong$ $Y \times k$, then $X \cong Y$ (see e.g. [1], [3, 4], [5], [6], 11]).

Let $X$ be an affine variety over $k$ with $\operatorname{dim} X \geq 7$. We show that there exists a smooth affine variety $X^{\prime}$, which is birationally equivalent to $X$, such that the variety $X^{\prime} \times k^{2}$ does not have $\mathrm{CP}$.

\section{Preliminaries}

Let $X$ be an affine variety (which is assumed to be irreducible) over $k$ and let $R=k[X]$ be the ring of regular functions on $X$ (by $k(X)$ we denote the field of rational functions on $X$ ). Let us recall some basic facts about algebraic vector bundles over $X$, which we identify with finitely generated projective $R$-modules. We say that an algebraic vector bundle $\mathbf{F}$ is stably trivial (of type $t$ ) if

$$
\mathbf{F} \oplus \mathbf{E}_{t}=\mathbf{E}_{s}
$$

Received by the editors December 15, 2008 and, in revised form, August 21, 2009 and December $9,2009$.

2010 Mathematics Subject Classification. Primary 14R10.

Key words and phrases. Algebraic vector bundle, cancellation problem.

The author was partially supported by a grant from the Polish Ministry of Science, 2010-2013.

(C)2010 American Mathematical Society

Reverts to public domain 28 years from publication 
for some trivial vector bundles $\mathbf{E}_{t}$ and $\mathbf{E}_{s}$. Recall that a vector $\left(f_{1}, \ldots, f_{r}\right) \in R^{r}$ is called a unimodular row if there exists a vector $g=\left(g_{1}, \ldots, g_{r}\right) \in R^{r}$ such that $\sum_{i=1}^{r} f_{i} g_{i}=1$. This is equivalent to the fact that $f_{1}, \ldots, f_{r}$ have no common zeros on $X$. A unimodular row $f=\left(f_{1}, \ldots, f_{r}\right) \in R^{r}$ determines uniquely a vector bundle $\mathbf{F}(f)=R^{r} / R g \cong\left(R^{r} / R f\right)^{*}$. Of course $\mathbf{F}(f) \oplus \mathbf{E}_{1}=\mathbf{E}_{r}$.

\section{Construction}

We start with the following nice classical example of Raynaud (see 13 for $k=\mathbb{C}$ and 14 for an arbitrary field $k$ ):

Example 3.1. Let $n \geq 3$ and

$$
R=\frac{k\left[x_{1}, \ldots, x_{n}, y_{1}, \ldots, y_{n}\right]}{\left(\sum_{i=1}^{n} x_{i} y_{i}-1\right)} .
$$

Then the stably free submodule of $R^{n}$ given by the unimodular row $\left(x_{1}, \ldots, x_{n}\right)$ is not free.

In the sequel the following result will be useful (for the proof see [8], Proposition 21):

Theorem 3.2. Let $X$ be an affine variety of dimension $n$ and let $Z \subset X$ be a closed subvariety. Take $m \geq n$. If the mapping $f: Z \rightarrow k^{m}$ is finite, then there exists a finite mapping $F: X \rightarrow k^{m}$ such that

$$
\left.F\right|_{Z}=f .
$$

We also need the following stronger version of a result from [7]:

Theorem 3.3. Let $X$ be a smooth affine variety and $Z \subset X$ a smooth closed subvariety. Assume that on $Z$ there is a stably trivial algebraic vector bundle $\mathbf{F}$ of type $t$. Then there is an open affine neighborhood $U \subset X$ of $Z$ and a stably trivial vector bundle $\mathbf{F}^{\prime}$ (of the same type $t$ ) on $U$ such that

$$
\left.\mathbf{F}^{\prime}\right|_{Z}=\mathbf{F} \text {. }
$$

In particular if $\mathbf{F}$ is not trivial, then neither is $\mathbf{F}^{\prime}$.

Proof. Let $\mathbf{F} \oplus \mathbf{E}_{t}=\mathbf{E}_{n}$. Hence $\mathbf{F}$ is the kernel of some epimorphism

$$
\phi: \mathbf{E}_{n} \ni\left(v_{1}, \ldots, v_{n}\right) \rightarrow\left(\sum_{i=1}^{n} f_{1 i} v_{i}, \ldots, \sum_{i=1}^{n} f_{t i} v_{i}\right) \in \mathbf{E}_{t},
$$

where $f_{i j} \in k[Z]$. Let $\mathbf{a}_{i}=\left(a_{i 1}, a_{i 2}, \ldots, a_{i n}\right) \in \mathbf{E}_{n}$ be sections such that $\phi\left(\mathbf{a}_{i}\right)=\mathbf{e}_{i}$, where $\mathbf{e}_{1}=(1,0, \ldots, 0), \ldots, \mathbf{e}_{t}=(0,0, \ldots, 1)$. Let $F_{i j} \in k[X]$ and $A_{i j} \in k[X]$ be extensions of the functions $f_{i j}$ and $a_{i j}$ to $X$. Take $\mathbf{A}_{i}=\left(A_{i 1}, \ldots, A_{i n}\right), i=1, \ldots, t$. Consider the following morphism of vector bundles on $X$ :

$$
\Phi: \mathbf{E}_{n} \ni\left(v_{1}, \ldots, v_{n}\right) \rightarrow\left(\sum_{i=1}^{n} F_{1 i} v_{i}, \ldots, \sum_{i=1}^{n} F_{t i} v_{i}\right) \in \mathbf{E}_{t} .
$$

Put

$$
U=\left\{x \in X: \operatorname{rank}\left\langle\Phi\left(\mathbf{A}_{\mathbf{1}}\right), \ldots, \Phi\left(\mathbf{A}_{\mathbf{t}}\right)\right\rangle=t\right\} .
$$

Note that $Z \subset U$. Moreover, on $U$ we have ker $\Phi \oplus\left\langle A_{1}, \ldots, A_{t}\right\rangle \cong \operatorname{ker} \Phi \oplus \mathbf{E}_{t}=\mathbf{E}_{n}$, i.e., ker $\Phi$ is a stably trivial vector bundle of type $t$ on $U$. Since $\left.\Phi\right|_{U}=\phi$ we have ker $\left.\Phi\right|_{U}=\operatorname{ker} \phi$. Put $\mathbf{F}^{\prime}=\operatorname{ker} \Phi$. 
Now it is enough to show that we can shrink $U$ to get $U^{\prime}$ affine. Indeed let $W=X \backslash U$. Then $W$ is closed and $W \cap Z=\emptyset$. If $n=\operatorname{dim} X$, then $\operatorname{dim} W, Z \leq$ $n-1$. In particular, there are finite mappings $f_{1}: W \rightarrow k^{n-1} \times\{1\} \subset k^{n}$ and $f_{0}: Z \rightarrow k^{n-1} \times\{0\} \subset k^{n}$. Since $W \cap Z=\emptyset$ we can glue these mappings to one map $f: Z \cup W \rightarrow k^{n}$. By Theorem 3.2 we can extend a finite mapping $f$ to a finite mapping $F: X \rightarrow k^{n}$. Now take $U^{\prime}=F^{-1}\left(k^{n} \backslash k^{n-1} \times\{1\}\right)$.

Now we are ready to prove:

Theorem 3.4. For every affine variety $X$ with $\operatorname{dim} X=n \geq 7$ there exists a smooth affine variety $Y$, which is birationally equivalent to $X$ and which possesses a stably trivial but not trivial algebraic vector bundle (of type 1 and rank 2).

Proof. We can assume that $X$ is smooth. Take a point $x \in X$. We can assume (after shrinking $X$ if necessary) that there are functions $\left\{f_{1}, \ldots, f_{n}\right\} \subset k[X]$, which form a system of local coordinates at $x$ and additionally $V\left(f_{1}, \ldots, f_{n}\right)=\{x\}$. Let us blow up the ideal $I=\left(f_{1}, \ldots, f_{n}\right)$. We obtain a quasiprojective variety $X^{\prime} \subset X \times \mathbb{P}^{n-1}$ birationally equivalent to $X$. Moreover, $X^{\prime}$ contains a subvariety $x \times \mathbb{P}^{n-1}$. Take $Y^{\prime}=X^{\prime} \cap\left(X \times k^{n-1}\right)$. Then $Y^{\prime}$ is an affine variety birationally equivalent to $X$ which contains a subvariety isomorphic to $k^{n-1}$. Since $n \geq 7$ we can embed into $Y^{\prime}$ a sphere $Z=\left\{(x, y) \in k^{6}: \sum_{i=1}^{3} x_{i} y_{i}=1\right\}$. By Example 3.1 on $Z$ there is a stably trivial but non-trivial algebraic vector bundle $\mathbf{F}$ (of type 1 and rank 2 ). By Theorem 3.3 we can extend this bundle to an open affine neighborhood $Y \subset Y^{\prime}$ of $Z$. Of course $Y$ is birationally equivalent to $Y^{\prime}$, hence also birationally equivalent to $X$.

We also have a more direct application of the Raynaud example:

Proposition 3.5. Let $X=\left\{(z, x, y) \in k^{m} \times k^{n} \times k^{n}: g\left(z_{1}, \ldots, z_{m}\right)=\sum_{i=1}^{n} x_{i} y_{i}\right\}$, where $g$ is a non-zero polynomial. Assume that $m>0$ and $n>2$. Then the algebraic vector bundle $\mathbf{F}$ with a smooth base $Y=X \backslash\{g=0\}$ given by the unimodular row $\left(x_{1}, \ldots, x_{n}\right)$ is stably trivial but non-trivial.

Proof. It is easy to check that $Y$ is a smooth variety. Now choose $z=z_{0}$ such that $g\left(z_{0}\right) \neq 0$. Then the bundle $\mathbf{F}$ restricted to the variety $X \cap\left(\left\{z_{0}\right\} \times k^{n} \times k^{n}\right)$ is the Raynaud vector bundle, hence it is non-trivial. Hence $\mathbf{F}$ is non-trivial, too.

Remark 3.6. Let us note that for $m=1$ and $g=z(z-1)$ we obtain the MohanKumar-Nori example; see [12].

\section{Applichtion to the CANCEllation problem}

In this section we apply our result to the cancellation problem. Let us recall the definition of a $k$-uniruled variety. First recall that a polynomial curve in $X$ is the image of the affine line $A^{1}(k)$ under a non-constant morphism $\phi: A^{1}(k) \rightarrow X$. Moreover, a family $\mathcal{F}$ of polynomial curves on $X$ is called bounded if there exists an embedding $i: X \subset k^{N}$ and a natural number $D$ such that every curve $\Gamma_{a} \in \mathcal{F}$ has degree less than or equal to $D$ in $k^{N}$. It is easy to see that the definition of bounded family does not depend on an embedding $i: X \rightarrow k^{N}$.

Now we have the following:

Definition 4.1 (see [9]). An affine variety $X$ is said to be $k$-uniruled if it is of dimension $\geq 1$ and there is a bounded family $\mathcal{F}$ of polynomial curves such that for every point $x \in X$ there is a curve $l_{x} \in \mathcal{F}$ going through $x$. 
The $k$-uniruled varieties can be characterized in a following way (see [9]):

Proposition 4.2. Let $X \subset k^{N}$ be an irreducible affine variety of dimension $\geq 1$. The following conditions are equivalent:

1) there is a bounded family $\mathcal{F}$ of affine polynomial curves, such that for every point $x \in X$ there is a curve $l_{x} \in \mathcal{F}$ going through $x$;

2) there is an open, non-empty subset $U \subset X$ and a bounded family $\mathcal{F}$ of affine polynomial curves such that for every point $x \in U$ there is a curve $l_{x} \in \mathcal{F}$ going through $x$;

3) there is an affine variety $W$ of dimension dim $X-1$ and a polynomial dominant mapping $\phi: W \times k \rightarrow X$.

We have the following important examples of non- $k$-uniruled varieties (see [7]):

Proposition 4.3. Let $h \in k\left[x_{1}, \ldots, x_{n}\right]$ be a non-constant polynomial. The variety

$$
X(h)=\left\{x \in k^{n}: h(x) \neq 0,1+x_{i}(h(x)-1) \neq 0 \text { for } i=1, \ldots, n\right\}
$$

is not $k$-uniruled.

Proof. Let $\phi: k \rightarrow X(h)$ be a regular mapping. Thus $\phi=\left(\phi_{1}(t), \ldots, \phi_{n}(t)\right)$, where $\phi_{i}$ are polynomials. Moreover, $h \circ \phi \neq 0$ for every $t$, which implies that $h \circ \phi$ is a non-zero constant. Similarly, $1+\phi_{i}(t)(h \circ \phi(t)-1)$ is a constant. Consequently, either $h \circ \phi(t)=1$ or all $\phi_{i}$ are constant. This means that outside the hypersurface $\left\{x \in k^{n}: h(x)=1\right\}$ there are no polynomial curves in $X(h)$.

In the sequel we need the following theorem, which was proved in [7:

Theorem 4.4. Let $X$ be a smooth affine non-k-uniruled variety with a stably trivial (of type 1) but non-trivial algebraic vector bundle $\mathbf{F}$ on it. If $\operatorname{rank} \mathbf{F}=r$, then the variety $X \times k^{r}$ fails $C P$.

Remark 4.5. In [7] the definition of $k$-uniruledness is slightly different than in our paper; however it is easy to check that all proofs in [7] work in our situation as well.

Now we are in a position to prove:

Theorem 4.6. Let $X$ be an affine variety of with $\operatorname{dim} X \geq 7$. There exists a smooth affine variety $X^{\prime}$ which is birationally equivalent to $X$ such that the variety $X^{\prime} \times k^{2}$ does not have $C P$.

Proof. By Theorem 3.4 there exists a smooth affine variety $Y$ which is birationally equivalent to $X$ and which possesses a stably trivial but non-trivial algebraic vector bundle $\mathbf{F}$ of type 1 and rank 2. Moreover, the bundle $\mathbf{F}$ is not trivial on some proper closed subset $Z$ of dimension smaller than $n=\operatorname{dim} Y$.

We show that we can find a Zariski open affine neighborhood $U$ of $Z$ which is non$k$-uniruled. Indeed, there is a finite mapping $f: Z \rightarrow k^{n-1} \times\{1\}$. By Theorem 3.2 we can extend this mapping to a finite map $F: Y \rightarrow k^{n}$.

Consider the variety

$$
X\left(x_{n}\right)=\left\{x \in k^{n}: x_{n} \neq 0,1+x_{i}\left(x_{n}-1\right) \neq 0 \text { for } i=1, \ldots, n\right\} .
$$

By Proposition 4.3 it is not $k$-uniruled. This also implies that the variety $X^{\prime}=$ $F^{-1}\left(X\left(x_{n}\right)\right)$ [which is birationally equivalent to $Y$, hence also to $X$ ] is not $k$ uniruled. By construction it contains $Z$; in particular the vector bundle $\mathbf{F}^{\prime}=\left.\mathbf{F}\right|_{X^{\prime}}$ is not trivial on $X^{\prime}$. Now by Theorem 4.4 the variety $X^{\prime} \times k^{2}$ does not have CP. 
Corollary 4.7. Let $K$ be a field which is finitely generated over $k$. Assume that $\operatorname{trdeg}(K / k)=n \geq 7$. Then there exists a smooth affine variety $W$ without $C P$, such that

$$
k(W)=K(x, y),
$$

where $x, y$ are elements algebraically independent over $K$.

Proof. Let $K=k\left(a_{1}, \ldots, a_{m}\right)$. Take $X=\operatorname{Spec}\left(k\left[a_{1}, \ldots, a_{m}\right]\right)$ and use Theorem 4.6.

Remark 4.8. It is worth noting that Theorem4.6 is in some sense the best possible. Indeed, if we start with a variety $X$, which has a non-uniruled projective closure, then every birational modification $X^{\prime}$ of $X$ is non- $k$-uniruled. In particular, $X^{\prime}$ and $X^{\prime} \times k$ do have CP (for details see [2]).

\section{REFERENCES}

[1] Danielewski, W., On a cancellation problem and automorphism groups of affine algebraic varieties, preprint, Warsaw (1989).

[2] Dryło, R., Non-uniruledness and the cancellation problem. II, Ann. Polon. Math., 92, 41-48 (2007). MR2318509 (2008c:14079)

[3] Dubouloz, A., Additive group actions on Danielewski varieties and the cancellation problem, Math. Z., 255, 77-93 (2007). MR2262722(2007i:14066)

[4] Fieseler, K., On complex affine surfaces with $\mathbb{C}_{+}$actions, Comment. Math. Helvetici, 69, 5-27 (1989). MR1259603 (95b:14027)

[5] Finston, D., Maubach, S., The automorphism group of certain factorial threefolds and a cancellation problem, Israel J. Math., 163, 369-381 (2008). MR.2391136 (2009a:14078)

[6] Fujita, T., On Zariski problem, Proc. Japan Acad., 55, 106-110 (1979). MR.531454 (80j:14029)

[7] Jelonek, Z., On the cancellation problem, Math. Ann., 344, 769-778 (2009). MR2507623

[8] Jelonek, Z., The set of points at which the polynomial mapping is not proper, Ann. Polon. Math., 58, 259-266 (1993). MR1244397(94i:14018)

[9] Jelonek, Z., Lasoń, M., The set of fixed points of a unipotent group, J. Algebra, 322, 21802185 (2009). MR 2542841

[10] Lam, T. Y., Serre Conjecture, Springer-Verlag, Berlin-Heidelberg, $1978 . \quad$ MR0485842 (58:5644)

[11] Miyanishi, M., Sugie, T., Affine surfaces containing cylinderlike open sets, J. Math. Kyoto Univ., 20, 11-42 (1980). MR.564667(81h:14020)

[12] Mohan Kumar, N., Stably free modules, Amer. J. of Math., 107, 1439-1443 (1985). MR815767 (87c:13014)

[13] Raynaud, M., Modules projectifs universels, Invent. Math., 6, 1-26 (1968). MR0236164 $(38: 4462)$

[14] Swan, R.G., Vector bundles, projective modules and the $K$-theory of spheres, Algebraic topology and algebraic K-theory (Princeton, NJ, 1983), 432-522, Ann. of Math. Stud., 113, Princeton Univ. Press, Princeton, NJ, 1987. MR921488 (89f:18009)

Instytut Matematyczny, Polska Akademia Nauk, Śniadeckich 8, 00-956 Warszawa, POLAND

E-mail address: najelone@cyf-kr.edu.pl 